\title{
Management of Diabetes in Pregnancy During COVID-19 Pandemic
}

\author{
Farhana Akter ${ }^{*}$, Adnan Mannan², Ershad Uddin Ahmed ${ }^{3}$ and A B M Kamrul-Hasan ${ }^{4}$ \\ ${ }^{1}$ Department of Endocrinology, Chittagong Medical College, Bangladesh
}

${ }^{2}$ Department of Genetic Engineering \& Biotechnology, University of Chittagong, Bangladesh

${ }^{3}$ Department of Gastroenterology, Chittagong Medical College, Bangladesh

${ }^{4}$ Department of Endocrinology, Mymensingh Medical College, Bangladesh

Submission: November 06, 2020; Published: December 17, 2020

*Corresponding author: Farhana Akter, Associate professor \& Head, Department of Endocrinology, Chittagong Medical College, Chattagram,4203, Bangladesh

\section{Introduction}

Pregnant women might be at an increased risk for severe illness from COVID-19 compared to non-pregnants. Additionally, there may be an increased risk of adverse pregnancy outcomes, such as preterm birth, among pregnant women with COVID-19 [1]. The anatomical structure of the respiratory system is changed during pregnancy, and the virus transmitted by droplets and aerosols is more easily inhaled by pregnant women and is difficult to remove [2]. Changes in reproductive hormones and immune systems during pregnancy collectively make them more susceptible to certain infections. More importantly, angiotensinconverting enzyme (ACE)-2, the SARS-CoV-2 receptor, has been proven highly increased during pregnancy, which may contribute to the susceptibility to SARS-CoV-2 [3].

\section{Covid-19 Pandemic and Pregnancy in Diabetes}

In the context of the COVID-19 pandemic, widespread anecdotal evidence suggests that both clinicians and pregnant women are increasingly unwilling to recommend or undergo the OGTT. This is based on valid concerns regarding travel, the possible need for two visits, and the time (up to $3 \mathrm{~h}$ ) spent in the potentially infectious environment of specimen collection centers. Moreover, a GDM diagnosis generally involves additional health service visits for diabetes education, glucose monitoring review, and fetal ultrasonography, all of which carry exposure risks during a pandemic [4]. During COVID-19 pandemic, there will be temporary changes to the recommended process of diagnostic testing for gestational diabetes during pregnancy and for postnatal checks in women who have had gestational diabetes [5]. The rationales for the suggestion of these temporary measures are shortening the screening test process to reduce risk of exposure to COVID-19 in laboratories; reducing the burden on pathology centers, diabetes care providers and obstetric team; minimizing the number of visits and duration of stay in the hospital for pregnant women; and reducing in-person visit by replacing such visits with remote communication with patients [2].

\section{Pregnancy Co-Morbidities and COVID-19 Infection}

It is important to consider the potential impact of pre-existing hyperglycemia and hypertension on the outcome of COVID-19 in pregnant women. Currently, there are higher risks of infection and poorer outcomes, including very high mortality, among the elderly population and those with co-morbidities such as hypertension and diabetes in this global pandemic. One in seven pregnancies is impacted by hyperglycemia and one in ten is impacted by hypertension. The stress of infection, accompanied by severe anxiety and use of high doses of corticosteroids, has the potential to worsen glycemic control and could enhance the risk of secondary infections [6].

\section{Epidemiology of COVID-19 and Pregnancy in Diabetes}

To date, no review has comprehensively evaluated the comparative data concerning pregnant and recently pregnant women and non-pregnant women with covid-19. Limitations in the external and internal validity of studies make it challenging for guideline developers and policy makers to make evidencebasedrecommendations for the management of pregnant and recently pregnant women with covid-19.The overall rate of covid-19 diagnosis in pregnant and recently pregnant women attending or admitted to hospital for any reason was 10\%, In the United States of America (USA) all available prevalence rates for covid-19 are greater than $15 \%$, one in 10 pregnant or recently pregnant women who are attending or admitted to hospital for any reason are diagnosed as having suspected or confirmed covid-19, 
although the rates vary by sampling strategy [7].

\section{Effects of COVID-19 on Pregnant mother and Neonate}

Most pregnant women will experience only mild or moderate cold/flu-like symptoms. Cough, fever, shortness of breath, headache and anosmia are other relevant symptoms [8]. Fever is common in COVID-19-infected patients. Previous data have demonstrated that maternal fever in early pregnancy can cause congenital structural abnormalities involving the neural tube, heart, kidney, and other organs [9-11]. It has been reported that viral pneumonia in pregnant women is associated with an increased risk of preterm birth, fetal growth restriction (FGR), and perinatal mortality [12]. The symptoms of severe infection are no different in pregnant women and early identification and assessment for prompt supportive treatment is the key. There is no evidence right now that COVID-19 causes problems with a baby's development or an increased risk of miscarriage. Few cases of neonatal COVID-19 infections have been reported but both were most likely an outcome of postnatal transmission [13].

\section{Vertical Transmission of Infection to Newborn of COVID-19 Suspected or Confirmed Mother}

At this point in the global pandemic of COVID-19 infection, currently there is no evidence for intrauterine vertical transmission of COVID-19 from infected pregnant mothers to their fetuses [14]. While this is reassuring, larger data are needed to firmly rule out trans-placental vertical transmission.

\section{Effects of Pregnancy on COVID-19}

Pregnancy is a physiological state that predisposes women to viral respiratory infection. Due to the physiological changes in the immune and cardiopulmonary systems, pregnant women are more likely to develop severe illness after infection with respiratory viruses. Pregnancy may also modify the clinical manifestation, for example lymphocytopenia may be even more marked [13].

Definition, Classification, Diagnosis, and Screening of Pregnancy in Diabetes

\section{Definition}

Diabetes in pregnancy may be either Pre-existing Diabetes or Gestational diabetes mellitus (GDM). GDM is defined as hyperglycemia with onset or first recognition during pregnancy, at severity less than those occur in overt diabetes [15]. GDM is a high-risk condition with adverse maternal and neonatal outcomes. Offspring of mothers with GDM are at risk for development of obesity and abnormal glucose metabolism during childhood, adolescence, and adulthood. Having fetal hyperinsulinism is a risk factor for development of both obesity and abnormal glucose metabolism, and might be implicated in pathophysiology $[16,17]$. In general, specific risks of diabetes in pregnancy include spontaneous abortion, fetal anomalies, preeclampsia, fetal demise, macrosomia, neonatal hypoglycemia, hyperbilirubinemia, and neonatal respiratory distress syndrome, among others $[18,19]$.

\section{Diagnosis and screening of GDM during COVID-19 pandemic [20]}

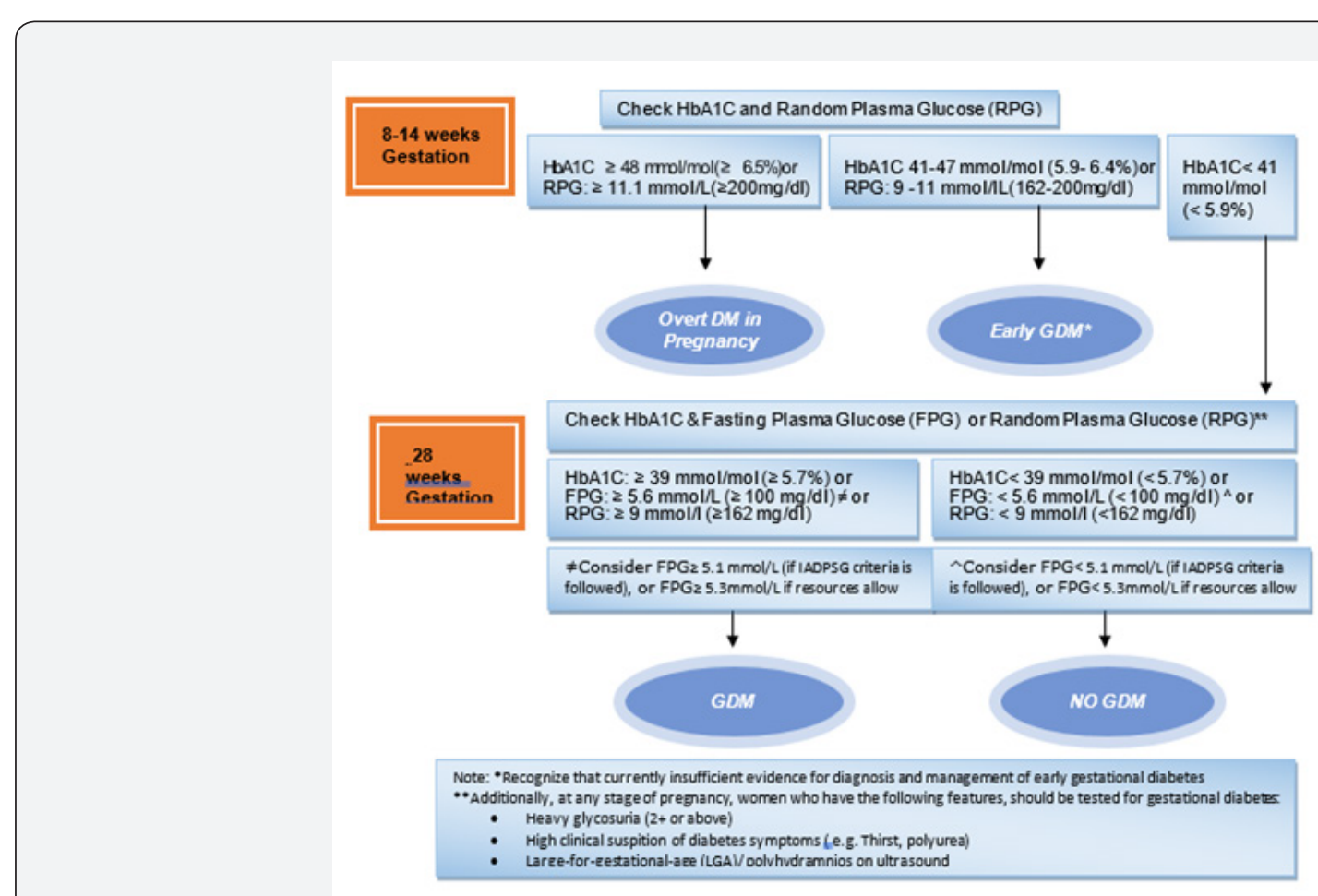

Figure 1: Screening for gestational diabetes mellitus in women with risk factors during the evolving COVID-19 pandemic. Figure adapted from the Royal College of Obstetricians and Gynecologists' Guidance for maternal medicine in the evolving coronavirus (COVID19) pandemic [21]. 
In an evolving pandemic with a highly infectious virus, screening OGTTs involve high exposure risks and health service burden. The routine use of OGTTs for GDM screening needs to be carefully considered in the context of local pandemic impact including community transmission rates. Where it is no longer safe or feasible current evidence does not support a single alternative test. We thus propose a strategy that utilizes alternative simpler tests and mitigation 'safety-nets' balancing GDM detection with minimizing of health service burden and viral exposure of women (Figure 1)

\section{Pre-Pregnancy Counseling}

According to recent guidelines from the American College of Obstetricians and Gynecologists, there is "no clear answer," as to whether couples should delay attempts to get pregnant. Individuals should make their own decisions based on their unique needs, desires, and values. Issues to consider include potential risks to pregnant individuals and their fetuses due to COVID-19, resource limitations, the social and financial effects of COVID-19 on an individual, and other factors [20]. So, this is an extremely personal decision, and couples should make upon consulting their physicians. At any time, but now more than ever, it is important for people to make an informed decision on whether to try to conceive. The most important point to realize is that there are still a lot of unknowns on how COVID-19 affects pregnancy, fetal development, and newborn babies. COVID-19 has only been around for several months. Mental health also plays an important role throughout this process. Women with preexisting diabetes should titrate insulin dose to reach target along with dietary readjustment and exercise as directed.

\section{Management of Diabetes in Pregnancy with Suspected or Conformed COVID-19}

\section{Lifestyle management}

After diagnosis, treatment starts with medical nutrition therapy, physical activity, and weight management depending on pre-gestational weight [4].

\section{Medical Nutrition Therapy}

Medical nutritional therapy is the keystone of treating GDM as it maintains desired glycemic goals in $80-90 \%$ of GDM women. The optimal dietary prescription would be a diet that provides adequate nutrition to support fetal and maternal well-being, while maintaining normoglycemia with absence of ketones, and achieving appropriate weight gain in pregnancy.

\section{Physical Exercise}

Exercise has been shown to improve glycemic control in GDM. Daily moderate exercise for 30 minutes or more is recommended for a woman with GDM, if she has no medical or obstetrics contraindications. Advising GDM patients to walk briskly or do arm exercises while seated in a chair for at least 10 minutes after each meal facilitates in reducing post-meal glucose rise and help in achieving glycemic goal. Patients can perform their exercise at home as far as possible, lockdown should not be an excuse to refrain from exercise.

\section{Smoking Cessation}

Smoking during pregnancy is the leading modifiable risk factor for poor birth outcomes, including stillbirth, miscarriage, and pre-term birth [21]. Smoking during pregnancy contributes to increased respiratory conditions among children and the World Health Organization has highlighted that exposure to secondhand smoke is a major cause of bronchitis, pneumonia, coughing and wheezing and asthma attacks in children [22]. Preliminary evidence indicates that smokers who contract the new coronavirus (COVID-19) have more severe symptoms [23]. One survey from China has found that smokers with COVID19 are 14 times more likely to develop severe disease [24]. It is vitally important that we help smokers to quit. Secondhand smoke exposure also elevates risks, so it is important that smokers are supported to maintain a smoke free home and pregnant women are not exposed to secondhand smoke in the home.

\section{Coping with Psychological Stress}

Pregnant women are at an increased risk for anxiety and depression; once they have been defined with suspected/ probable/confirmed COVID-19 infection they may exhibit varying degrees of psychiatric symptoms that are detrimental to maternal and fetal health [25]. Mother/baby separation may impede early bonding as well as establishment of lactation [26]. These factors will inevitably cause additional stress for mothers in the postpartum period. Healthcare providers should pay attention to a patient's mental health, including promptly assessing her sleep patterns and sources of anxiety, depression, and even suicidal ideation. A perinatal psychiatrist should be consulted when necessary.

\section{Pharmacological Intervention}

If the medical nutrition therapy and exercise fail to achieve glycemic goals for a woman with GDM, insulin therapy should be initiated. Insulin therapy is the primary pharmacological treatment. The type and timing of insulin should be chosen based on the specific blood glucose elevation [27].

\section{Antenatal care: Antenatal visits, Physical checkup, and laboratory tests}

All women diagnosed with GDM should have an appointment with the diabetes midwife/nurse, who will provide training in the use of a glucose meter. Where feasible, this should be done remotely via video call. This visit should also be used as an opportunity to provide women with dietetic information and contact details of the dietician, where one is available. Women should be followedup remotely in the week after the meter training by the diabetes midwife/nurse and for all appointments where home capillary blood sugar levels are to be checked by the diabetes team. 
Women should be provided with clear guidance on who to contact if they have $>3$ abnormal blood glucose levels in a week or $>10-15 \%$ of all readings - this will usually be the diabetes antenatal team [28]. Antenatal care for pregnant women with GDM should, wherever possible, minimize the need for hospitalbased care, support public health measures concerning physical distancing and self-isolation and reduce health service burden. Retinal screening services should be targeted for the highest risk women (proliferative and previously treated proliferative retinopathy, pre- proliferative retinopathy, or maculopathy), and those without retinal screening in past 2 years [29].

Specific Management of Suspected, Probable, or Confirmed cases of COVID-19 in Pregnancy

Patient should be managed initially by designated tertiary hospitals with effective isolation facilities and protection equipment. Suspected/probable cases should be treated in isolation and confirmed cases should be managed in a negative pressure isolation room, although it is recognized that these may be unavailable in many units. In general, critically ill confirmed cases should be admitted to a negative pressure isolation room in an ICU [30]. If negative pressure isolation rooms are not available, patients should be isolated in single rooms or grouped together once COVID-19 infection has been confirmed. Chest CT scanning has high sensitivity for diagnosis of COVID-19 [31]. In a pregnant woman with suspected COVID-19 infection, a chest CT scan may be considered as a primary tool for the detection of COVID-19 in epidemic areas. Informed consent should be obtained, and a radiation shield applied over the gravid uterus.

\section{Maternal Surveillance}

Maintain fluid and electrolyte balance; symptomatic treatment, such as antipyretic, anti-diarrheal medicines. Close and vigilant monitoring of vital signs and oxygen saturation level to minimize maternal hypoxia; conduct arterial blood-gas analysis; repeat chest imaging (when indicated); regular evaluation of complete blood count, renal and liver function testing, and coagulation testing. If antiviral treatment is to be considered, this should be done following careful discussion with virologists; pregnant patients should be counseled thoroughly on the potential adverse effects of antiviral treatment for the patient herself as well as for the risk of FGR.

Monitoring for bacterial infection (blood culture, midstream or catheterized specimen urine microscopy and culture) should be done, with timely use of appropriate antibiotics when there is evidence of secondary bacterial infection. Severe pneumonia is associated with a high maternal and perinatal mortality rate, therefore aggressive treatment is required, including supporting measures with hydration and oxygen therapy. Consideration for the use of low molecular weight heparin in severe cases; however, its efficacy in improving the outcomes of severe COVID-19 pneumonia requires further investigation before formal recommendation [6].

\section{Fetal surveillance}

Undertake cardiotocography (CTG) for fetal heart rate (FHR) monitoring when gestational age is beyond the limit of viability based on local practice (23-28 weeks).

Glycemic targets and self-monitoring of blood glucose (SMBG)

The level of glycemic target in pregnancy is shown in Table 1 [32].

Table 1: Glycemic targets during diabetes in pregnancy.

\begin{tabular}{|c|c|}
\hline Plasma Glucose/HbA1C & Target \\
\hline Fasting plasma glucose & $<95 \mathrm{mg} / \mathrm{dL}(5.3 \mathrm{mmol} / \mathrm{L})$ \\
\hline AND EITHER & \\
\hline One-hour postprandial glucose & $<140 \mathrm{mg} / \mathrm{dL}(7.8 \mathrm{mmol} / \mathrm{L})$ \\
\hline OR & \\
\hline Two-hour postprandial glucose & $<120 \mathrm{mg} / \mathrm{dL}(6.7 \mathrm{mmol} / \mathrm{L})$ \\
\hline HbA1C & $<6 \%$ \\
\hline
\end{tabular}

\section{Blood glucose monitoring}

Women are instructed to carry out self-monitoring of blood glucose (SMBG) 4 times a day like, fasting glucose (upon awakening), and one- or 2-hour post-meals (after the first bite of a meal) [28].

\section{Intrapartum management during labour and delivery}

All pregnant women with or recovering from COVID-19 should be provided with counseling and information related to the potential risk of adverse pregnancy outcomes. Institutional delivery should be preferred over home delivery in these women. The use of birthing pools during labor should be avoided in confirmed or suspected cases of COVID-19, given the potential risk of infection via feces [29]. Mode of birth should be individualized, based on obstetric indications and the woman's preferences. WHO recommends that induction of labour and caesarean section should only be undertaken when medically justified and based on maternal and fetal condition? Suspected COVID-19 infection is not an indication for cesarean section, unless the woman's respiratory condition demands urgent delivery, or pregnant women have other indications. If caesarean section is indicated, the procedure should be performed in a negative pressure isolation operating room.

\section{Postpartum management and neonatal care}

\section{Postpartum management of woman}

Mother/baby separation may impede early bonding as well as establishment of lactation. These factors will inevitably cause additional stress for mothers in the postpartum period. Unless there is a reasonable suspicion of postpartum type 2 diabetes, postpartum follow-up testing, most likely using an OGTT, should be delayed until either the pandemic has been controlled or 
another pregnancy is contemplated [4].

\section{Management of newborn}

Regarding neonatal management of suspected, probable, and confirmed cases of maternal COVID-19 infection, the umbilical cord should be clamped promptly, and the neonate should be transferred to the resuscitation area for assessment by the attending pediatric team. There is insufficient evidence regarding whether delayed cord clamping increases the risk of infection to the newborn via direct contact [33]. In units in which delayed cord clamping is recommended, clinicians should consider carefully whether this practice should be continued [34].

\section{Covid -19 and Breast feeding}

Relatively few cases have been reported of infants confirmed with COVID-19; those that have been reported experienced mild illness. There is currently insufficient evidence regarding the safety of breastfeeding and the need for mother/baby separation. Except in the sickest women with COVID-19 infections (i.e., those requiring respiratory support or intensive care admission), the benefits of breast feeding outweigh any potential transmission risks [29]. Parents should be informed that infection with COVID-19 is not a contraindication to breastfeeding. A risks and benefits discussion with neonatologists and families is recommended to individualize care in babies who may be more susceptible. Some recommendations by WHO regarding Breast feeding advice during covid-19 pandemic:

A. When mother is suspected or confirmed Covid -19 positive, she can follow the steps:

-Use a medical mask when you near to her child.

-Wash hands thoroughly with soap or sanitizer before and after contact with your child

-Routinely clean and disinfect any surfaces you touch

B. If mother is severely ill with covid-19 or suffer from other complications that prevent her from caring of her infant or continuing direct breastfeeding, express milk to safely provide breastmilk to her infant.

C. If mother is too unwell to breastfeed or express breastmilk, use another approach, one that is acceptable and available to her.

Relactation: Restarting breastfeeding after a gap

Wet Nursing: Other women breastfeeding or caring for her child

Donor Human Milk: Whenever or not the mother or child has suspected, probable, or confirmed covid-19, healthcare staffs should enable mothers and infant to: :

- $\quad$ Remain together
- $\quad$ Practice skin-to-skin contact and

- Room-in throughout the day and night, especially straight afterbirth during establishment of breast feeding

\section{Contraception after delivery during Covid-19}

Long-acting reversible contraceptives - IUDs and implants - are highly effective and safe and may be considered first-line for women with gestational diabetes. If access to postnatal long-acting reversible contraception is limited, postpartum medroxyprogesterone (Depo-Provera) injections are offered before hospital discharge [29].

\section{Conclusion}

We suggest pragmatic options for screening, diagnosis, and management of diabetes in pregnancy during the pandemic with emphasis on risk-stratified approaches and in the context of local practice and facilities available. Management strategies should focus on changes to care prompted by the pandemic.

\section{References}

1. Coronavirus Disease 2019 (COVID-19): Considerations for Inpatient Obstetric Healthcare Settings. Centers for Disease Control and Prevention.

2. Nouhjah S, Jahanfar S, Shahbazian H (2020) Temporary changes in clinical guidelines of gestational diabetes screening and management during COVID-19 outbreak: A narrative review. Diabetes \& Metabolic Syndrome: Clinical Research \& Reviews 14: 939-942.

3. Zhao X, Jiang Y, Zhao Y, Xi H, Liu C, et al. (2020) Analysis of the susceptibility to COVID-19 in pregnancy and recommendations on potential drug screening. European Journal of Clinical Microbiology \& Infectious Diseases 39(7): 1209-1220.

4. McIntyre HD, Moses RG (2020) The Diagnosis and Management of Gestational Diabetes Mellitus in the Context of the COVID-19 Pandemic. Diabetes Care 43: 1433-1434.

5. Australasian Diabetes in Pregnancy Society, Australian Diabetes Society, Australian Diabetes Educators Association, Diabetes Australia. Diagnostic testing for gestational diabetes mellitus (GDM) during the COVID 19 pandemic: antenatal and postnatal testing advice.

6. International Federation Gynecology and Obstetrics (FIGO). NonCommunicable Diseases and COVID-19: Statement from the FIGO Committee on Pregnancy and NCDs.

7. Allotey J, Stallings E, Bonet M, Yap M, Chatterjee S, et al. (2020) Clinical manifestations, risk factors, and maternal and perinatal outcomes of coronavirus disease 2019 in pregnancy: living systematic review and meta-analysis. BMJ 370

8. Coronavirus (COVID-19) Infection in Pregnancy. Royal College of Obstetricians \& Gynecologists

9. Vallejo V, Ilagan JG (2020) A Postpartum Death Due to Coronavirus Disease 2019 (COVID-19) in the United States. Obstet Gynecol 136(1): 52-55.

10. Hantoushzadeh S, Shamshirsaz AA, Aleyasin A, Ashraf Aleyasin, Maxim D Seferovic, et al. (2020) Maternal Death Due to COVID-19 Disease. American journal of obstetrics and gynecology 223(1): 109. 
11. Karami P, Naghavi M, Feyzi A, Mehdi Aghamohammadi, Mohammad Sadegh Novin, et al. (2020) Mortality of a pregnant patient diagnosed with COVID-19: A case report with clinical, radiological, and histopathological findings. Travel Med Infect Dis 101665.

12. Henderson J, Gao H, Redshaw M (2013) Experiencing maternity care: the care received and perceptions of women from different ethnic groups. BMC Pregnancy Childbirth 13(1): 196.

13. Poon LC, Yang H, Kapur A, Melamed N, Dao B, et al. (2020) Global interim guidance on coronavirus disease 2019 (COVID-19) during pregnancy and puerperium from FIGO and allied partners: Information for healthcare professionals. Int J Gynecol Obstet 149(3): 273-286.

14. Zarchia MK, Neamatzadeh H, Dastgheib SA, Abbasi H, Mirjalilid SR, et al. (2020) Vertical Transmission of Coronavirus Disease 19 (COVID-19) from Infected Pregnant Mothers to Neonates: A Review. Fetal and Pediatr Pathol 39(3): 246-250.

15. Metzger BE (2010) International association of diabetes and pregnancy study groups recommendations on the diagnosis and classification of hyperglycemia in pregnancy. Diabetes Care 33(3): 676-682.

16. Hillier TA, Pedula KL, Schmidt MM, Mullen JA, Charles MA, et al. (2007) Childhood obesity and metabolic imprinting: the ongoing effects of maternal hyperglycemia. Diabetes Care 30(9): 2287-2292.

17. Metzger BE (2007) Long-term outcomes in mothers diagnosed with gestational diabetes mellitus and their offspring. Clin Obstet Gynecol 50(4): 972-979.

18. Dabelea D, Hanson RL, Lindsay RS, D J Pettitt, G Imperatore, et al. (2000) Intrauterine exposure to diabetes conveys risks for type 2 diabetes and obesity: a study of discordant sibships. Diabetes 49(12): 2208-2211.

19. Holmes VA, Young IS, Patterson CC, Donald W M Pearson, James D Walker, et al. (2011) Optimal glycemic control, preeclampsia, and gestational hypertension in women with type 1 diabetes in the Diabetes and Pre-eclampsia Intervention Trial. Diabetes Care 34(8): 1683-1688.

20. Thangaratinam S, Cooray SD, Sukumar N, Huda MSB, Devlieger R, et al. (2020) Endocrinology in the time of COVID-19: Diagnosis and management of gestational diabetes mellitus. Eur J Endocrinol 183(2): G49-G56.

21. Royal College of Obstetricians and Gynaecologists (2020) Guidance for Maternal Medicine in the Evolving coronavirus (COVID-19) Pandemic - Information for Healthcare Professionals.
22. American College of Obstetricians and Gynecologists (ACOG). COVID-19 FAQs for obstetricians-gynecologists, gynecology: Should individuals delay attempts to become pregnant during COVID-19 pandemic?

23. Smoking in Pregnancy Challenge Group (2018) Review of the Challenge.

24. WHO (1999) International Consultation on Environmental Tobacco Smoke (ETS) and Child Health. Consultation Report.

25. Vardavas C, Nikitara K (2020) COVID-19 and Smoking: A systematic review of the evidence. Tobacco Induced Diseases.

26. Dorheim SK, Bjorvatn B, Eberhard-Gran M (2012) Insomnia and depressive symptoms in late pregnancy: A population-based study. Behav Sleep Med 10(3): 152-166.

27. Chua M, Lee J, Sulaiman S, Tan HK (2020) From the frontlines of COVID-19- How prepared are we as obstetricians: A commentary. BJOG 127(7): 786-788.

28. Alfadhli EM (2015) Gestational diabetes mellitus. Saudi Med J 36(4): 399-406.

29. Murphy HR (2020) Managing Diabetes in Pregnancy Before, During, and After COVID-19. Diabetes Technology \&Therapeutics 22(6): 454 461.

30. Maxwell C, Mc Geer A, Tai KFY, Sermer M (2017) No 225-Management guidelines for obstetric patients and neonates born to mothers with suspected or probable severe acute respiratory syndrome (SARS). J Obstet Gynaecol Can39: e130-e137.

31. Zhao W, Zhong Z, Xie X, Yu Q, Liu J (2020) Relation between chest CT findings and clinical conditions of coronavirus disease (COVID-19) pneumonia: A multicenter study. AJR Am J Roentgenol 214(5): 10721077.

32. American Diabetes Association (2020) Management of diabetes in pregnancy: standards of medical care in Diabetes-2020. Diabetes Care; 43(Suppl.1): S183-S192.

33. Yang H, Wang C, Poon LC (2020) Novel coronavirus infection and pregnancy. Ultrasound Obstet Gynecol 55(4): 435-437.

34. World Health Organization. Breastfeeding advice during the COVID-19 outbreak.

\section{Your next submission with Juniper Publishers} will reach you the below assets

- Quality Editorial service

- Swift Peer Review

- Reprints availability

- E-prints Service

- Manuscript Podcast for convenient understanding

- Global attainment for your research

- Manuscript accessibility in different formats

( Pdf, E-pub, Full Text, Audio)

- Unceasing customer service

Track the below URL for one-step submission https://juniperpublishers.com/online-submission.php 